\title{
Quality Inspection of Fruits and Vegetables using Colour Sorting in Machine Vision System: A review
}

\author{
ANISH.P ${ }^{1}$, GOKUL RAM.M² \\ 1. Sri Ramakrishna Engineering College \\ 2. Sri Ramakrishna Engineering College
}

\section{ABSTRACT:}

Quality is an important aspect in the trading and business of vegetable and fruits. The customer satisfaction and acceptance depends greatly on the external appearance of these food items. The qualities assessed include the size, shape, colour and texture. At present only manual inspection is being done to brand the quality of the product. Manual inspection is challenging in terms of the speed of inspection and the reliability of the output. Machine vision systems may be used as an object of quality evaluation. Various methods such as colour sorting and pattern recognition can be used to evaluate the quality of the fruit or vegetable. Machine vision systems provide a nondestructive, reliable, accurate and fast results regarding the external appearance of the fruit or vegetable. The application of lighting, cameras and image processing are being discussed here with an eye on the future developments possible.

Keywords: Colour Sorting, Pattern Recognition, non-destructive, image-processing

\section{INTRODUCTION:}

Fruits and Vegetables form a very essential and irreplaceable part of the animal food cycle. The external appearance of these fruits and vegetables decides the market rates and the sales of these items ( Costa et al., 2011) [5]. In some cases when the quality of the fruits are not upto the mark, i.e. they are contaminated or are defective in nature, they can lead to the spread of infections among the people and also leading to economic problems ( ElMasry, Wang, Vigneault, Qiao, \& ElSayed, 2008) [10]. Therefore, external quality inspection is mandatory and extremely necessary ( Teena, Manickavasagan, Mothershaw, El Hadi, \& Jayas, 2013) [27].

Automatic external quality inspection of fruits and vegetables is still a work under progress. Some external quality criteria, such as colour, texture, size, and shape, are actually standardised on industrial graders, but the grading of fruits and vegetables according to the other appearance criteria, such as rottenness, and some other unknown defects which present the same colour and texture to the peel is not yet efficient and consequently remains a manual operation ( Leemans \& Destain, 2004) [19]. Manual operation has some disadvantages such as inconsistency, time consumption and variability in results. Also, the manual process is very tedious, laborious, costly, and easily influenced by the surrounding environment ( ElMasry, Cubero, Molto, \& Blasco, 2012; Elmasry, Kamruzzaman, Sun, \& Allen, 2012; Razmjooy, Mousavi, \& Soleymani, 2012) [8] [9] [26]. So it is necessary to develop an automatic external quality inspection system to replace manual inspection. 
Machine vision is an engineering technology that combines mechanics, optical instrumentation, electromagnetic sensing, digital video and image processing technology (Patel, Kar, Jha, \& Khan, 2012) [23]. And it is the area of engineering which deals with the study and application of methods which enable the computer to understand the content of an image, and this interpretation involves the extraction of certain characteristics which are important for a given aim ( Gomes \& Leta, 2012) [12]. Over the past few years the development of information science, image processing and pattern recognition technology has been rapid. Computer hardware and software and computer vision has been developed as an inspection tool for quality of a variety of food products. In the automatic external quality inspection, this technology aims to duplicate the effect of the human vision by electronically perceiving and understanding an image ( Brosnan \& Sun, 2002) [3], recognize and interpret the external characters of fruits and vegetables, and provide the information for the external quality sorting and grading machine.

The most common type of computer vision system used for external quality inspection is the traditional computer vision system which is based on RGB colour cameras that imitate the vision of the human eyes by capturing images using three filters centred at red (R), green (G) and blue (B) wavelengths ( Lorente et al., 2012) [21]. Most of the external quality characteristics, such as colour, texture, size, and shape can be detected by using this system. But for some unknown defects, it is difficult to detect by the usage of the traditional computer vision system due to its lack of spectral information in conventional colour images. A typical spectral image is composed of a set of monochromatic images corresponding to certain wavelengths, and hyperspectral and multispectral computer vision systems have the natural advantage compared to the traditional computer vision. The extensive spectral and imaging information acquired by the hyperspectral and multispectral computer vision systems makes it possible to decode some appearance features that are impossible or difficult with the traditional system. Many applications have been studied using this multispectral and hyperspectral computer vision systems. Therefore it is expected to be an exceptional tool to study fruit and vegetable external quality characteristics.

Several review papers have been published ( Davies, 2009; Feng \& Sun, 2012; Liu, Zeng, \& Sun, 2013; Qin, Chao, Kim, Lu, \& Burks, 2013) [6] [11] [20] [25], some of these papers are only based on traditional computer vision system. The others are focused on hyperspectral and multispectral imaging in quality (external, internal and safety) evaluation. Therefore, the main objectives of this paper are to give a summarization of the different types of computer vision systems and explain their basic principles, latest developments and applications of computer vision in external quality inspection of fruits and vegetables. Additionally, the principle components, basic theories, and corresponding processing and analysis methods are also reported.

\section{CONSTRUCTION OF MACHINE VISION SYSTEM}

Fig. 1 shows the structure of a typical machine vision system. As shown in Fig. 1 a machine vision system generally consists of the following five basic components: illumination, a camera, a frame grabber and computer hardwareand software ( Wang \& Sun, 2002) [28]. Additionally, a spectrograph which disperses the wavelength and a transportation stage are additional components of the hyperspectral or multispectral machine vision systems. 


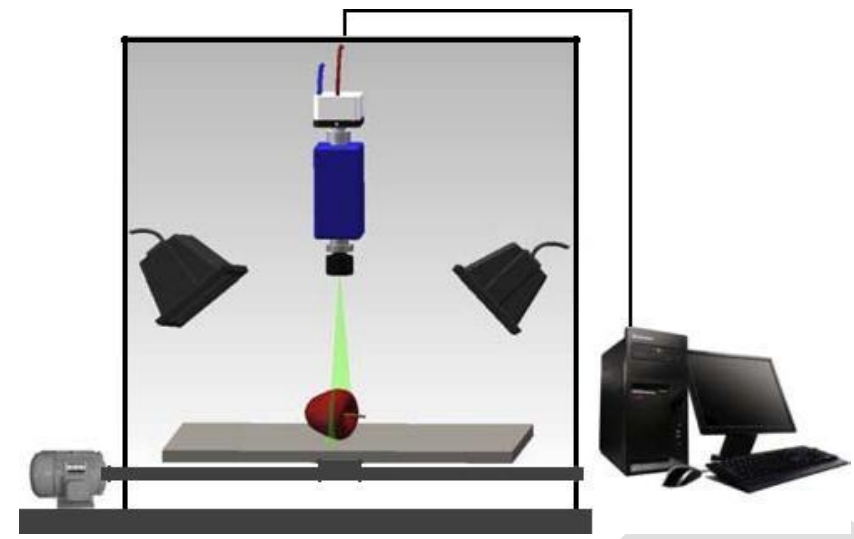

Fig. 1. A schematic of a typical computer vision system. *Just for hyperspectral and multispectral computer vision systems.

Illumination is a very important component of the machine vision system. Similar to the human eyes, machine vision systems are affected by the level and quality of illumination. Illumination devices generate light that illuminates the desired target objects, therefore the quality of the illumination system can greatly influence the quality of images and plays an important role in the accuracy of the system (Novini \& Engineers, 1995) [22]. Good illumination reduces the shadow and reflection and simultaneously enhances the contrast of the image. In the external quality inspection of fruits and vegetables using machine vision system, the illumination takes place through two methods; front lighting and back lighting. Front lighting (electron projection lithography or reflective illumination) is mainly used in conditions where the surface quality characteristics are to be inspected such as colour, texture and also the skin defects. The back lighting (transmitted illumination) arrangement is used in situations where the edge or boundary quality characteristics such as size and shape are to be inspected. The positions of the component, types of lamps, and colour quality required are all factors when choosing the most suitable illumination ( Teena et al., 2013) [27]. Incandescent lamps, fluorescent lamps, lasers, and infrared lamps are the commonly used light sources ( Kodagali, 2012) [17].

The camera is one of the major components in machine vision systems. Common image acquisition systems used include magnetic resonance imaging (MRI), ultrasound technology, computed tomography (CT) and electrical tomography (Du \& Sun, 2004) [7]. Charged Coupled Device (CCD) and Complementary Metal Oxide Semiconductor (CMOS) image sensors are the two different methods that can be used to generate the image digitally. A machine vision system consisting of a high resolution CCD camera and its associated hardware is one of the most widely used inspection systems in the external quality inspection of fruits and vegetables. Array or area scan type cameras consist of a matrix of minute photosensitive elements (photo sites) from which the entire image of the object is obtained based on output proportional to the amount of incident light ( Brosnan \& Sun, 2002) [3]. However the line scan type cameras consist of only a single line of photo sites, only a line image of the object is obtained at one time, therefore a stage of movement or transportation is used to move the given sample under the camera to achieve the complete image line by line.

The frame grabber is an electronic device in machine vision system that is usually used to capture the individual, digital still frames from video cameras, and then display and store in raw or compressed digital form. 
The computer hardware and software, which resembles the human brain, is another key component of machine vision system. In the external quality of fruits and vegetables, the computer hardware and software processes and analyses the image and gives the final inspection information about the object.

Wavelength dispersion devices form the core components of the hyperspectral and multispectral machine vision systems. They are used for dispersing light into different wavelengths and projecting the transmitted light to an area detector. Prism, gratings and filter are some of the widely used wavelength dispersion devices. The principles of prism and diffraction grating are illustrated in Fig. 2. All these types of dispersion devices can be attached to a lens and area camera to form a line- or area-scan spectral camera system. Generally, prism and diffraction grating are used in the hyperspectral machine vision system, and filters are widely used in the multispectral machine vision system.
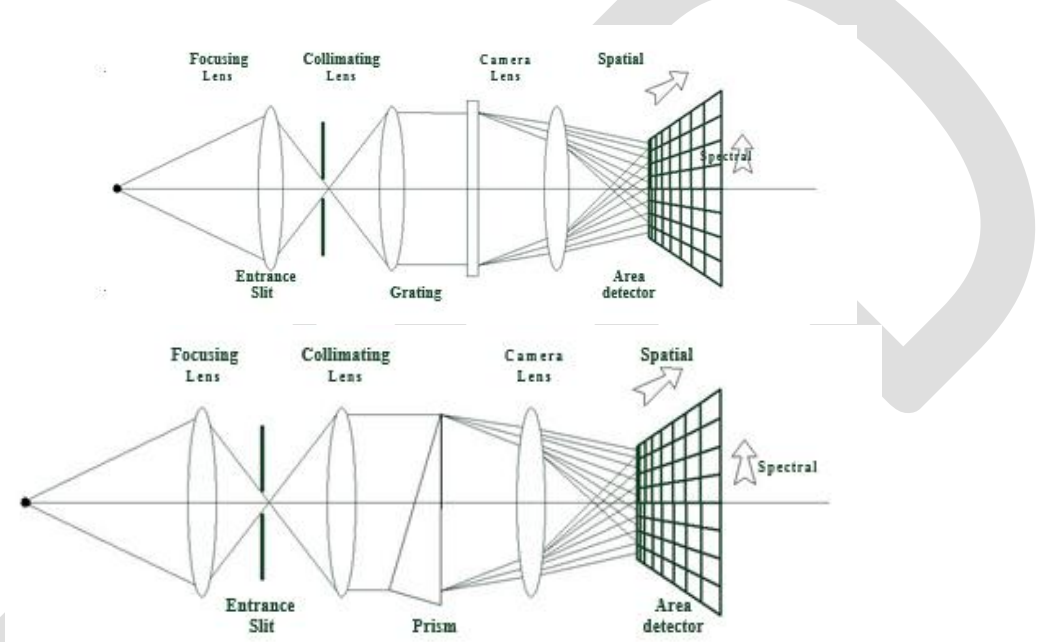

Fig. 2. Operating principles of diffraction grating and prism.

\section{MACHINE VISION SYSTEMS:}

Traditional, hyperspectral and multispectral machine vision systems are the three most widely used types of vision systems in the external quality inspection of fruits and vegetables. Every system has its advantages and disadvantages. The brief histories, principles, advantages and disadvantages of three different machine vision systems will be given in the following sections.

\subsection{Traditional computer vision system}

Traditional machine vision is a young discipline of engineering with its origin traced back to the 1960s. Following a sudden increase of interest during the 1970s, it has experienced continued growth in both theory and application ( Baxes, 1994; Patel et al., 2012) [2] [23]. Nowadays, traditional machine vision system is widely used in medical imaging, industrial automation, security monitoring, military applications, aerospace field, intelligent transportation system, food quality and safety inspection, autonomous vehicle and robot guidance, etc.

The human eyes are mainly sensitive to the primary colours red, green and blue. Hence the traditional machine vision system is normally based on RGB colour cameras that imitate the vision of the eyes by capturing images using three filters centred at the wavelengths of red, green and blue (RGB) ( Lorente et al., 2012) [21]. So the images captured by RGB colour cameras are very close to the actual visuals perceived by the human eyes in colour. Fig. 3 shows a colour image of a peach, and its $\mathrm{R}, \mathrm{G}$ and $\mathrm{B}$ component monochromatic images. 
Most external quality attributes, such as colour, texture, size, shape, and some obvious defects, can be inspected and graded automatically with traditional machine vision system. But because of the lack of spectral and multi-constituent information in traditional colour images, some other unobvious defects, whose colour and texture are closely similar to the normal skin, are still difficult to detect automatically only by using the traditional system.

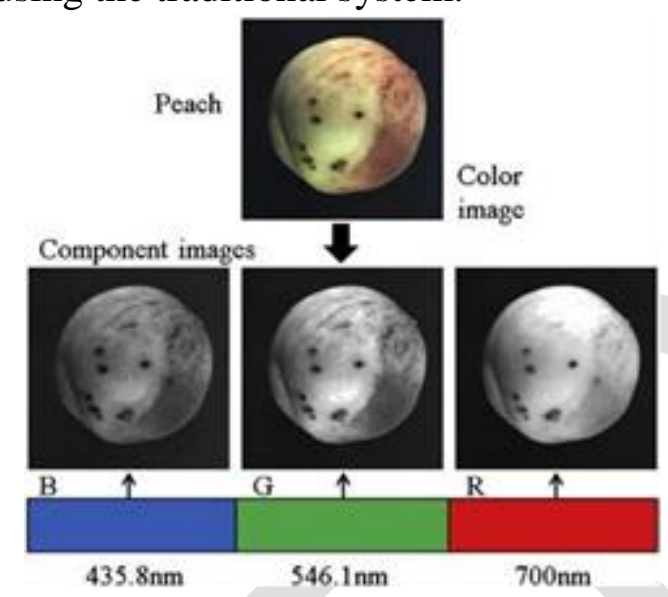

Fig. 3. A RGB color image of a peach and its component images.

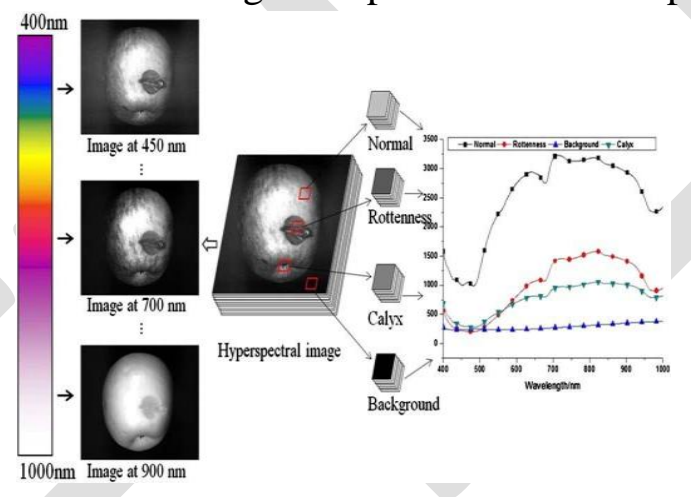

Fig. 4. Conceptual view of a hyperspectral image of an apple with rottenness.

\subsection{Hyperspectral computer vision system}

Unlike the traditional machine vision system, which can only capture the three monochromatic images centred at RGB wavelengths, hyperspectral machine vision system, or hyperspectral imaging system, conjoins both spectroscopic and imaging techniques into one system to get a set of monochromatic images at almost continuous wavelengths. Therefore, the integrated system can provide spatial information, the same as conventional imaging system, along with spectral information, the same as spectroscopic devices, for every individual pixel of the spatial image. Fig. 4 shows the conceptual view of a hyperspectral image of an apple with rottenness.

\subsection{Multispectral computer vision system}

Multispectral machine vision system, or multispectral imaging system, is different from hyperspectral imaging system in the number of the monochromatic images in the spectral domain. Multi-spectral imaging, generally, is a form of imaging that involves capturing two or more different waveband monochromatic images in the spectrum. The traditional RGB camera could be considered to be similar to as a particular case of a multispectral imaging system. But unlike the traditional digital camera method of capturing the specific frequency light that falls on the installed detector, 
multispectral machine vision system helps us to combine two or more single-band monochromatic images. Fig. 5 shows the conceptual view of a multispectral image of a peach with spot defects.

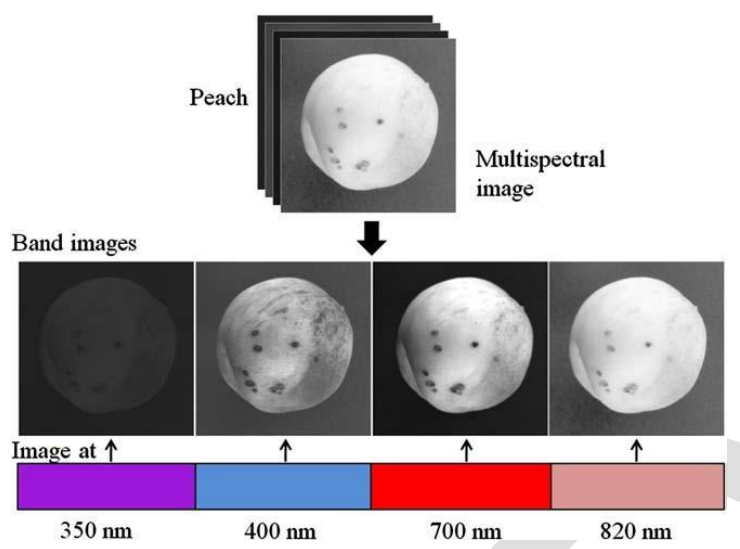

Fig. 5. Conceptual view of a multispectral image of a peach with spot defects.

\section{HYPERSPECTRAL IMAGE PROCESSING AND ANALYSIS TECHNIQUES}

The processing and analysis of hyperspectral images are different from that of RGB images from the traditional systems or monochromatic images of the multispectral systems. Based on the data of the hyperspectral image, a hyperspectral image can be considered to be either a set of twodimensional spatial monochromaticimages at each individual wavelength of the full spectrum, or a group of spectrums at each individual pixel of the image. Image correction and selection of efficient wavelength are the most essential steps in the processing and analysis of hyperspectral images. Image correction can ensure the stability of the hyperspectral machine vision system, and efficient wavelength selection is the important step to realize the in-line inspection by shortening the image acquiring and processing times.

\subsection{Image correction}

Hyperspectral computer vision system is commonly carried out in one of the three following modes: reflectance, transmittance or scatter modes. In the external quality inspection of fruits and vegetables, the reflectance mode is the most widely used acquisition mode. The final hyperspectral image of the fruits or vegetables is obtained and recorded in accordance with the hyperspectral imaging system.

To improve the comparability of the hyperspectral image data, the hyperspectral image must be transformed into the reflectance mode. This can be done using the combination of dark reference image and the radiance of light source. ( Gomez-Sanchis, Gomez-Chova et al., 2008; GomezSanchis, Molto et al., 2008) [13][15].

\subsection{The efficient wavelength selection}

Hyperspectral machine vision system provides extensive information about the inspection targets which in this case are fruits and vegetables. In the basic researches, the hyperspectral imaging acquired and the algorithm obtained from inspection are very time-consuming due to the large amount of data. The hyperspectral image is highly correlated. Only some of the monochromatic images in the full spectrum are efficient in the external quality inspection tasks. To realize the in-line inspection, the transformation must be done from the fundamental research level of hyperspectral imaging to the fast application level of multispectral imaging. Selection of the most efficient 
wavelengths for specific task of inspection is the key and challenging step to complete this transformation.

Principle Component Analysis is a very effective data reduction and efficient wavelength selection technique for the hyperspectral images. Hyperspectral wavelengths are transformed into sequence of component bands (PC bands) which are the linear combinations of the original bands by the PCA. The largest percentage of the original information are contained in the first several PC band images. Therefore the fact that the first several PC band images can illustrate main features present in the hyperspectral image is understood. ( Kim et al., 2002) [16].

\section{IMAGE PROCESSING AND ANALYSIS TECHNIQUES}

The core of the machine vision refers to the image processing and image analysis with various algorithms and methods available to complete the specific classification and measurement (Brosnan \& Sun, 2004; Kurtz et al., 2000) [4] [18]. Image processing and analysis can be performed in three levels as illustrated in Fig. 6. The low level processing or the basic processing of image, involves image acquisition and image pre-processing; the intermediate level processing or the make-or-break step in image processing and analysis, involves image segmentation, feature extraction, representation, and description; the high level processing, which is one of the key steps of image analysis, involves recognition, interpretation and classification.

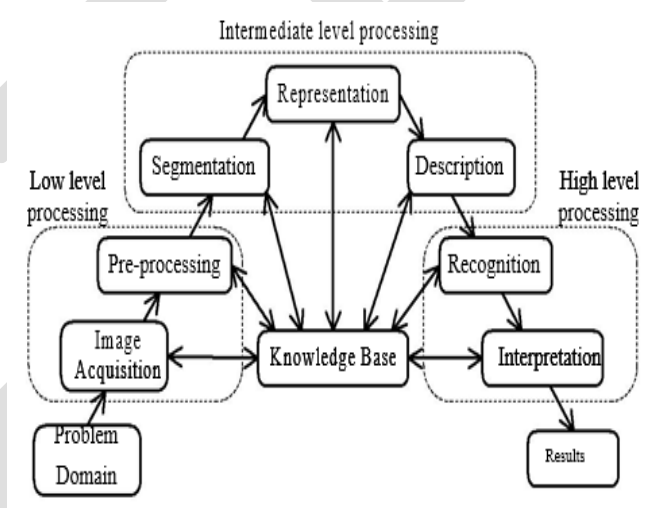

Fig. 6. Different levels of image processing ( Du \& Sun, 2004).

\section{APPLICATIONS OF MACHINE VISION IN THE EXTERNAL QUALITY INSPECTION OF FRUITS AND VEGETABLES}

All types of machine vision systems, including the traditional, hyperspectral and multispectral systems, maybe widely used in the fruit and vegetable industry for inspecting the external quality features and grading in terms of the appearance of the product. Colour is one of the most important sensorial quality attributes of fruits and vegetables based on which their sales in the market is determined. Colour of fruits and vegetables mainly depends on the internal biochemical and chemical changes which occur in growth, ripeness. Therefore colour inspection has been used as the indirect measurement of some internal quality attributes,such as maturity, freshness, variety and desirability, and safety ( Pathare, Opara, \& Al-Said, 2013; Wu \& Sun, 2013) [24] [29]. Colour is the highest elementary information that is stored in pixels, and it contains the basic visual information in the images corresponding to human vision ( Zheng, Sun, \& Zheng, 2006) [30]. 


\section{CHALLENGES AND FUTURE TRENDS}

Machine vision systems have become a common and extremely useful scientific tool in industrial and agricultural automation due to superior performance, ongoing improvements in cost and ease of use. ( Teena et al., 2013) [27]. However, to achieve the fault detection more quickly and accurately, there are still many challenges to be overcome. Some of these challenges include recognition of stem-calyx, the irregular distribution of lightness on curvature surface, whole surface inspection, long time consumption for processing the spectral image, efficient selection of the wavelengths, and discrimination of the different defects.

Also, major advances in 3D techniques, Terahertz imaging, X-ray, and Raman imaging can be used as a substitute to machine vision in the quality inspection of fruits and vegetables.

\section{CONCLUSION}

Various appearance characters, including colour, texture, size, shape, and variety of defects, are the most important external quality attributes of fruits and vegetables. Machine vision systems, including traditional computer vision system, hyperspectral computer vision system, and multispectral computer vision system, have been widely used in the quality inspection of fruits and vegetables to replace manual inspection as they can provide a fast, accurate and non-destructive testing. Many successful examples have proved that the machine vision systems are powerful tools for the accurate, quick and automatic external quality inspection of fruits and vegetables. This paper reviews the principle, the procedure, developments and applications of three machine vision systems in the external quality inspection of fruits and vegetables. There are still many challenges to overcome, as a promising technology, machine vision will continuously play an important role in the researches and applications for the quality inspection of fruits and vegetables.

\section{REFERENCES}

[1] Abdullah, M. Z., Mohamad-Saleh, J., Fathinul-Syahir, A. S., \& Mohd-Azemi, B. M. N. (2006). Discrimination and classification of fresh-cut starfruits (Averrhoa carambola L.) using automated machine vision system. Journal of Food Engineering, 76(4), 506-523

[2] Baxes, G. A. (1994). Digital image processing: Principles and applications.: Wiley.

[3] Brosnan, T., \& Sun, D. -W. (2002). Inspection and grading of agricultural and food products by computer vision systems-A review. Computers and Electronics in Agriculture, 36(2), 193-213.

[4] Brosnan, T., \& Sun, D. W. (2004). Improving quality inspection of food products by computer vision - A review. Journal of Food Engineering, 61(1), 3-16.

[5] Costa, C., Antonucci, F., Pallottino, F., Aguzzi, J., Sun, D. W., \& Menesatti, P. (2011). Shape analysis of agricultural products: A review of recent research advances and potential application to computer vision. Food and Bioprocess Technology, 4(5), 673-692.

[6] Davies, E. R. (2009). The application of machine vision to food and agriculture: A review. Imaging Science Journal, 57(4), 197-217.

[7] Du, C. -J., \& Sun, D. -W. (2006). Learning techniques used in computer vision for food quality 
evaluation: A review. Journal of Food Engineering, 72(1), 39-55.

[8] ElMasry, G., Cubero, S., Molto, E., \& Blasco, J. (2012). In-line sorting of irregular potatoes by using automated computer-based machine vision system. Journal of Food Engineering, 112(1-2), 60-68.

[9] Elmasry, G., Kamruzzaman, M., Sun, D. W., \& Allen, P. (2012). Principles and applications of hyperspectral imaging in quality evaluation of agro-food products: A review. Critical Reviews in Food Science and Nutrition, 52(11), 999-1023.

[10] ElMasry, G., Wang, N., ElSayed, A., \& Ngadi, M. (2007). Hyperspectral imaging for nondestructive determination of some quality attributes for strawberry. Journal of Food Engineering, 81(1), 98-107.

[11] Feng, Y. Z., \& Sun, D. W. (2012). Application of hyperspectral imaging in food safety inspection and control: A review. Critical Reviews in Food Science and Nutrition, 52(11), 10391058.

[12] Gomes, J. F. S., \& Leta, F. R. (2012). Applications of computer vision techniques in the agriculture and food industry: A review. European Food Research and Technology, 235(6), 9891000.

[13] Gomez-Sanchis, J., Gomez-Chova, L., Aleixos, N., Camps-Valls, G., Montesinos-Herrero, C., Molto, E., et al. (2008). Hyperspectral system for early detection of rottenness caused by Penicillium digitatum in mandarins. Journal of Food Engineering, 89(1), 80-86.

[14] Gomez-Sanchis, J., Martin-Guerrero, J. D., Soria-Olivas, E., Martinez-Sober, M., MagdalenaBenedito, R., \& Blasco, J. (2012). Detecting rottenness caused byPenicilliumgenus fungi in citrus fruits using machine learning techniques. Expert Systems with Applications, 39(1), 780-785.

[15] Gomez-Sanchis, J., Molto, E., Camps-Valls, G., Gomez-Chova, L., Aleixos, N., \& Blasco, J. (2008). Automatic correction of the effects of the light source on spherical objects. An application to the analysis of hyperspectral images of citrus fruits. Journal of Food Engineering, 85(2), 191-200.

[16] Kim, D. G., Burks, T. F., Qin, J., \& Bulanon, D. M. (2009). Classification of grapefruit peel diseases using color texture feature analysis. International Journal of Agricultural and Biological Engineering, 2(3), 41-50.

[17] Kodagali, J. (2012). Computer vision and image analysis based techniques for automatic characterization of fruits: A review. International Journal of Computer Applications, 50(6), 6.

[18] Kurtz, M. J., Eichhorn, G., Accomazzi, A., Grant, C., Murray, S. S., \& Watson, J. M. (2000). The NASA astrophysics data system: Overview. arXiv preprint astro-ph/0002104.

[19] Leemans, V., \& Destain, M. F. (2004). A real-time grading method of apples based on fea- tures extracted from defects. Journal of Food Engineering, 61(1), 83-89.

[20] Liu, D., Zeng, X. -A., \& Sun, D. -W. (2013). Recent developments and applications of 
hyperspectral imaging for quality evaluation of agricultural products: A review. Critical Reviews in Food Science and Nutrition (just-accepted).

[21] Lorente, D., Aleixos, N., Gomez-Sanchis, J., Cubero, S., Garcia-Navarrete, O. L., \& Blasco, J. (2012). Recent advances and applications of hyperspectral imaging for fruit and vegetable quality assessment. Food and Bioprocess Technology, 5(4), 1121-1142.

[22] Novini, A. R., \& Engineers, S. o. M. (1995). The latest in vision technology in today's food and beverage container manufacturing industry: Society of Manufacturing Engineers.

[23] Patel, K. K., Kar, A., Jha, S. N., \& Khan, M. A. (2012). Machine vision system: A tool for quality inspection of food and agricultural products. Journal of Food Science and TechnologyMysore, 49(2), 123-141.

[24] Pathare, P. B., Opara, U. L., \& Al-Said, F. A. (2013). Colour measurement and analysis in fresh and processed foods: review. Food and Bioprocess Technology, 6(1), 36-60.

[25] Qin, J. W., Chao, K. L., Kim, M. S., Lu, R. F., \& Burks, T. F. (2013). Hyperspectral and multispectral imaging for evaluating food safety and quality. Journal of Food Engineering, 118(2), 157171.

[26] Razmjooy, N., Mousavi, B. S., \& Soleymani, F. (2012). A real-time mathematical computer method for potato inspection using machine vision. Computers \& Mathematics with Applications, 63(1), 268-279.

[27] Teena, M., Manickavasagan, A., Mothershaw, A., El Hadi, S., \& Jayas, D. S. (2013). Potential of machine vision techniques for detecting fecal and microbial contamination of food products: A review. Food and Bioprocess Technology, 6(7), 1621-1634.

[28] Wang, H. H., \& Sun, D. W. (2002). Correlation between cheese meltability determined with a computer vision method and with Arnott and Schreiber tests. Journal of Food Science, 67(2), 745749.

[29] Wu, D., \& Sun, D. W. (2013). Colour measurements by computer vision for food quality control - A review. Trends in Food Science \& Technology, 29(1), 5-20.

[30] Zheng, C. X., Sun, D. W., \& Zheng, L. Y. (2006). Recent developments and applicationsof image features for food quality evaluation and inspection - A review. Trends in Food Science \& Technology, 17(12), 642-655. 